\title{
Breast Cancer Classification Using Deep Convolutional Neural Networks
}

\author{
${ }^{1}$ Jennifer K. Chukwu, ${ }^{1}$ Faisal S. Bala and ${ }^{* 2}$ Aliyu S. Nuhu \\ ${ }^{1}$ Department of Mechatronics and Systems Engineering, Abubakar Tafawa Balewa University, Bauchi, Nigeria \\ ${ }^{2}$ Department of Electrical and Electronics Engineering, University of Jos, Jos, Nigeria \\ jnyfaah@gmail.com | faisalsani@ymail.com I nuhua@unijos.edu.ng
}

Received: 11-FEB-2021; Reviewed: 02-MAR-2021; Accepted: 13-APR-2021

http://dx.doi.org/10.46792/fuoyejet.v6i2.617

\begin{abstract}
Breast cancer remains the primary causes of death for women and much effort has been depleted in the form of screening series for prevention. Given the exponential growth in the number of mammograms collected, computer-assisted diagnosis has become a necessity. Histopathological imaging is one of the methods for cancer diagnosis where Pathologists examine tissue cells under different microscopic standards but disagree on the final decision. In this context, the use of automatic image processing techniques resulting from deep learning denotes a promising avenue for assisting in the diagnosis of breast cancer. In this paper, an android software for breast cancer classification using deep learning approach based on a Convolutional Neural Network (CNN) was developed. The software aims to classify the breast tumors to benign or malignant. Experimental results on histopathological images using the BreakHis dataset shows that the DenseNet CNN model achieved high processing performances with $96 \%$ of accuracy in the breast cancer classification task when compared with state-ofthe-art models.
\end{abstract}

Keywords- Breast cancer classification, Convolutional Neural Network (CNN), deep learning, DenseNet, histopathological images

\section{INTRODUCTION}

A ccording WHO statistics, over 100,000 Nigerians are diagnosed with cancer annually, and about 80,000 die from the disease, averaging 240 Nigerians every day or 10 Nigerians every hour, dying from cancer (Ogundipe, 2017). Breast cancer is a key public health issue because it is the most mutual cancer in women and the leading cause of cancer death worldwide. Indeed, nearly one in seven women will be affected by this pathology during its existence, the risk growing with age (Cireşan et al, 2013). The development of massive breast cancer screening has led to earlier diagnosis and rapid management with a significant improvement in survival rate. The treatment and analysis of medical images is swiftly expanding area where the problem of automatically searching for information contained in medical images is instantly needed.

The great diversity of medical imaging devices, the difficulty in interpreting these images as well as their large number, generates wearisome work for those who must interpret them (Ahmad, 2018). In order to process this large volume of information, doctors are currently turning to the use of systems to assist in the analysis and interpretation of these images. This analysis aims to facilitate the diagnosis made by the practitioner and to make it as accurate and reliable as possible (Platania et al., 2018). Among the various machine learning techniques, the deep neural networks are attracting remarkable interest due to their automatic feature extraction and representation learning ability they are considered a significant development in technology as it has displayed performance beyond the existing machine learning tasks including object detection and classification (Cireşan et al, 2013).

\footnotetext{
${ }^{*}$ Corresponding Author

Section B- ELECTRICAL/COMPUTER ENGINEERING \& COMPUTING SCIENCES Can be cited as:

Chukwu J., Bala F.S., and Nuhu A.S. (2021): Breast Cancer Classification Using Deep Convolutional Neural Networks, FUOYE Journal of Engineering and $\begin{array}{llll}\text { Technology } & \text { (FUOYEJET), } & \text { 6(2), 35-38. }\end{array}$ http://dx.doi.org/10.46792/fuoyejet.v6i2.617
}

Contrary to conventional machine learning methods, which require a hand-crafted feature extraction stage which is challenging as it relies on domain knowledge, deep learning methods adaptively learn the appropriate feature extraction process from the input data with respect to the target output. This eliminates the tedious process of engineering and explicitly programming the system, deep learning is far superior as it can learn from raw data.

Deep learning refers to advanced statistical learning methods organized in multiple layers, to extract representations of data on multiple levels, and whose layers are not predefined by the user but learned directly from the data by the algorithm thus mirroring human neuronal functioning (Irshad, 2014). The main challenge of cancer automatic aided diagnosis systems is dealing with the intrinsic complexity of histopathological images. To deal with this, a powerful convolutional neural network of binary classification was chosen. DenseNet model was proposed, one of state of the art in the image recognition competition ImageNet (Irshad, 2014). The DenseNet is built for natural images processing but it was modified with histopathology images for breast cancer classification using transfer learning.

\section{ReLATEd Work}

Tao et al. (2007) proposed a breast mass classification from mammograms using deep convolutional neural networks (CNN). They presented how CNN can be used to directly classify pre-segmented breast masses using AlexNet. Lévy et al. (2016) introduced breast cancer mass classification from mammograms using deep CNN. They evaluated some preliminary experiments using the deep learning approach to classify breast cancer images with AlexNet. Nawaz et al. (2016) also presented a classification of breast cancer histology images using AlexNet. They proposed a transfer learning-based approach for the classification of breast cancer images.

Bayramoglu (2016) proposed deep learning for breast cancer histopathology image classification. The system classifies breast cancer images independent of their 
magnifications using Single-task and multi-task CNN. Similarly, Han et al. (2017) proposed to apply a fastscanning deep CNN to pixel-wise region segmentation using a customized Architecture. Ahmad et al. (2018) experimented with classification of breast cancer histology images using transfer learning trained and evaluated four (4) state of the art CNN architectures (AlexNet, GoogLeNet/Inception, VGGNet and RNN) using mammographic datasets.

\section{Proposed CNN Model for Breast Cancer CLASSIFICATION}

A Convolutional Neural Network (CNN) is feedforward neural network introduced by Kunihiko Fukushima in 1980 and improved by Yann LeCan et al. in 2001. A CNN is composed of 6 types of layers: an input layers, a convolutional layer, a non-linear layer, a pooling layer, fully connected layer, and an output layer. Figure 1 illustrates a traditional CNN architecture.

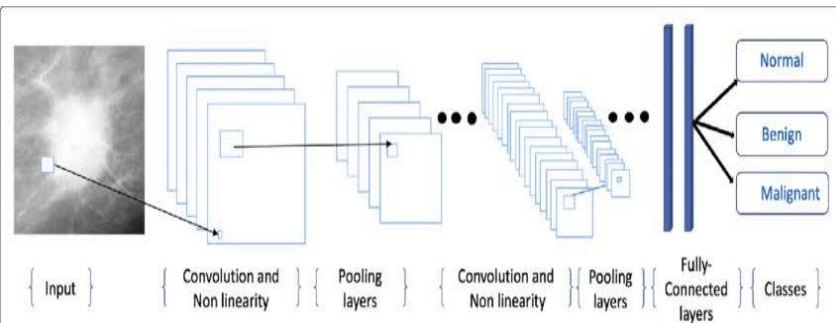

Fig. 1: Convolutional neural network

The pre-trained Convolutional Neural Network model, i.e. Dense Convolutional Network (Dense-Net) was used for feature extraction process via transfer learning, a machine learning method where a model developed for a task is re-used as the starting point for a model on a second task. This model is a logical extension of Residual Convolutional Network (ResNet). It is a new CNN architecture that reached the State-Of-The-Art (SOTA) results on classification datasets (CIFAR, SVHN, ImageNet) using less parameters. To facilitate downsampling in our architecture we divide the network into multiple densely connected dense blocks as shown in Figure 2.

A Dense-Net is composed of dense blocks. In those blocks, the layers are densely connected together. This means that each layer receives input from all preceding layers and passes its own information to all subsequent layers, in this case the final output layer has direct information from every single layer as this helps improve the problem of redundant layers.

To reduce the size, DenseNet uses transition layers. These layers contain convolution with kernel size $=1$ followed by $2 \times 2$ average pooling with stride $=2$. It reduces height and width dimensions but leaves feature dimension the same. The input is an image with shape $(28,28,48)$, which resulted to an output image with shapes $(14,14,48)$. The DenseNet scale naturally to hundreds of layers, while exhibiting no optimization difficulties. Thus, that makes DenseNet one of the most powerful models in image recognition tasks.

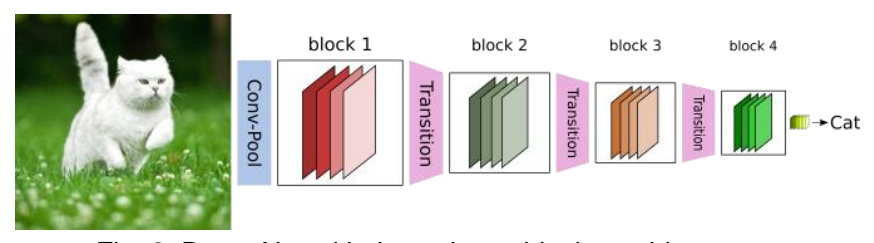

Fig. 2: DenseNet with three dense blocks architectures (Irshad, 2014)

Transfer learning is defined as fine-tuning CNN models pre-trained from natural image dataset to medical image tasks. Learning from clinical images is usually difficult due to its computational cost, convergence problem, and insufficient number of high quality labelled samples. A growing body of experiments has investigated pretrained models in the presence of limited learning samples. An initialized weights of different layers of the proposed network was carried out using pre-trained model on ImageNet. Then, we employed last layer finetuning on BreakHis cancer images dataset. Therefore, ImageNet pretrained weights were preserved while the last fully connected layer was updated continuously. The first convolutional layer of the network is then un-frozen, and the entire network is fine-tuned on the BreakHis training data. The advantage of the DenseNet is feature concatenation that helps the system to learn the features in any stage without the need to compress them and the ability to control and manipulate those features. This technique helps to reduce the training process complexity and eliminate the over fitting problem.

\section{EXPERIMENTS}

Appropriate datasets are required at all stages of object classification research, starting from training phase to evaluating the performance of classification algorithms. The Breast Cancer Histopathological Image Classification (BreakHis) dataset was used for this project. It is composed of 9,109 breast tumour tissue microscopic images. The researchers collected samples from 82 patients, and used different magnifying factors (40x, 100x, 200x, and 400x) (Spanhol, F. A et al., 2016). The image distribution is summarized in Table 1.

Table 1. Image Distribution in the BREAKHIS Dataset

\begin{tabular}{|c|c|c|c|c|}
\hline Class & $40 x$ & $100 x$ & $200 x$ & $400 x$ \\
\hline Benign & 625 & 644 & 623 & 588 \\
\hline Malignant & 1370 & 1437 & 1390 & 1232 \\
\hline Total & 1995 & 2081 & 2013 & 1820 \\
\hline
\end{tabular}

The samples are stained with hematoxylin and eosin. The author of the dataset uses breast tissue biopsy slides to generate these samples while pathologists from the P\&D lab labeled them. Figure 3 shows the breast tumor specimens assessed by Immunohistochemistry. The biopsy procedure was Surgical Open Biopsy. An Olympus BX-50 system microscope was used to capture the images. As aforementioned, they images were captured using four magnification factor, 40x, 10x 200x 
and 400x. The images were in Portable Network Graphics (PNG) format, in 3-channel RGB, 8-bit depth.
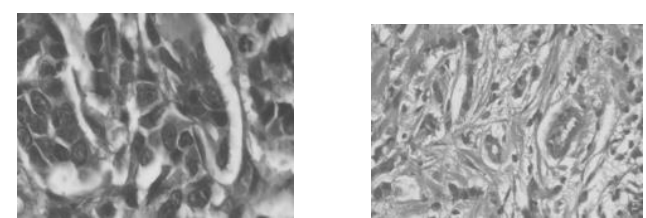

Fig. 3: Same tumor under different magnification

To develop the model, we use the Tensorflow deep learning framework and the keras. The model is trained and tested using the Hp series desktop equipped with an Intel i7 processor and an Nvidia Geforce GTX960 GPU. Following the experimental protocol proposed in Irshad, $\mathrm{H}$, (2014)., the dataset is divided into $70 \%$ for training set and $30 \%$ for validation set. When discussing medical images, there are two ways to report the results. In the first one the decision is patient-wise; therefore, the recognition rate is computed at the patient level. Let $\mathrm{Np}$ be the number of histopathological images of patient $P$. For each patient, if $\mathrm{N}$ cancer images are correctly classified, the patient score and the global patient recognition rate are defined as in equation 1 and equation 2 respectively. In the second case the recognition rate is computed at the image level. Let $\mathrm{Nt}$ be the number of histopathological images of the testing set. If $\mathrm{Nr}$ cancer images are correctly classified, then the recognition rate at the image level is represented in (3).

$$
\text { Patient Score }=\frac{N}{N_{p}}
$$

$$
\begin{gathered}
\text { Patient Recognition Rate }=\frac{\sum \text { Patient Score }}{\text { Total number of Patient }} \\
\text { Image Recognition Rate }=\frac{N_{r}}{N_{t}}
\end{gathered}
$$

The proposed CNN model aims to treat the highresolution images generally used for the histopathological classification of breast cancer. The DenseNet model is modified to extract fully global feature from the histological images and use them in the training process using transfer learning. In this case we resize all the images to $224 \times 224 \times 3$ RGB color space. After obtaining the weights of the model pre-trained on ImageNet, transfer learning is done in the following steps. First, the fully connected layer has randomly initialized weights.

We freeze the convolutional layers of the network, and only train the fully connected layer using the BreakHis training dataset. The fully connected layer is trained from scratch on the features extracted from the fixed convolutional layers. The first convolutional layer of the network is then unfrozen, and the entire network is finetuned on the BreakHis training data. This involves retraining the $\mathrm{CNN}$, starting from the retained weights, and using a very small step size. To train the model we use the Adam optimizer to minimize the loss function. Adam optimizer is a gradient descent algorithm with an adaptive momentum that computes adaptive learning rates for each parameter. Figure 4 represents the total loss minimization during the training process. The training process took 11 hours and the total loss achieves a minimum of 0.3424 .

\section{Result Discussion}

After training the proposed model, the dataset was reserved for validation. Table 2 reports the accuracy of the model with the different magnification factors of the BreakHis dataset in both image level and patient level. The proposed model reported about $96 \%$ accuracy on the image level. Histopathology tumor detection and classification into classes would play a key role in breast cancer diagnosis, reduce the heavy workloads of pathologists and establish the appropriate therapeutic approach by doctors. It achieves, respectively, 95.4\% of average accuracy of the image level and $96.48 \%$ accuracy of the patient level for all magnification factors.

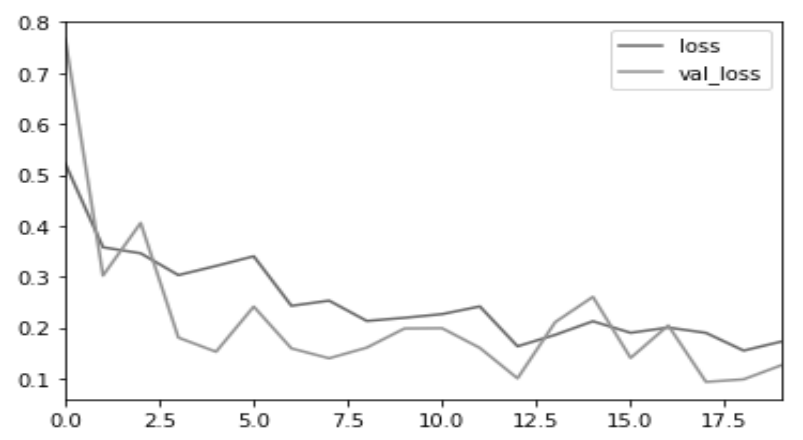

Fig. 4: Plot of validation loss and training loss

Table 2. The Model Classification Accuracy

\begin{tabular}{|c|c|c|c|c|c|}
\hline $\begin{array}{c}\text { Magnification } \\
\text { factor }\end{array}$ & $40 \mathrm{X}$ & $100 \mathrm{X}$ & $200 \mathrm{X}$ & $400 \mathrm{X}$ & Average \\
\hline $\begin{array}{c}\text { Image Level } \\
\text { Accuracy (\%) }\end{array}$ & 93.64 & 97.42 & 95.87 & 94.67 & 95.4 \\
\hline $\begin{array}{c}\text { Patient Level } \\
\text { Accuracy (\%) }\end{array}$ & 94.23 & 97.86 & 96.35 & 95.25 & 96.48 \\
\hline
\end{tabular}

After testing, to further provide a proper performance evaluation, the result of our model was compared with powerful CNN models for histopathological breast cancer image classification. The state-of-the-art models' comparison with the proposed model is presented in Table 3. The model was finally converted to tflite format and deployed on android mobile as shown in the Figure 5 .

Table 3. Model Comparison with some Popular CNN Breast Cancer Classification

\begin{tabular}{|c|c|c|c|c|c|c|}
\hline \multirow{2}{*}{$\begin{array}{c}\text { Accuracy } \\
(\%)\end{array}$} & Model & \multicolumn{5}{|c|}{ Magnification } \\
\cline { 3 - 7 } & $\begin{array}{c}\text { LeNet } \\
\text { (Lecun, } \\
2001)\end{array}$ & 40.4 & 47.34 & 46.5 & 45.2 & 46.36 \\
\hline \multirow{7}{*}{$\begin{array}{c}\text { Image } \\
\text { level }\end{array}$} & $\begin{array}{c}\text { AlexNet } \\
\text { (Kumar, } \\
\text { 2016) }\end{array}$ & 86.4 & 75.8 & 72.6 & 84.6 & 79.85 \\
\cline { 2 - 7 } & $\begin{array}{c}\text { CSDCNN } \\
\text { (Ahmad, } \\
2018)\end{array}$ & 92.8 & 93.9 & 93.4 & 92.9 & 93.25 \\
\cline { 2 - 7 } & $\begin{array}{c}\text { DenseNet } \\
\text { (Proposed) }\end{array}$ & 93.64 & 97.42 & 95.87 & 94.67 & 95.4 \\
\hline \multirow{7}{*}{$\begin{array}{c}\text { Patient } \\
\text { level }\end{array}$} & LeNet & 48.2 & 47.6 & 45.5 & 45.2 & 46.62 \\
\cline { 2 - 7 } & AlexNet & 74.6 & 73.8 & 76.4 & 79.2 & 76 \\
\cline { 2 - 7 } & CSDCNN & 94.1 & 93.2 & 94.7 & 93.5 & 93.87 \\
\cline { 2 - 7 } & $\begin{array}{c}\text { DenseNet } \\
\text { (Proposed) }\end{array}$ & 94.23 & 97.86 & 96.35 & 95.24 & 96.48 \\
\hline
\end{tabular}




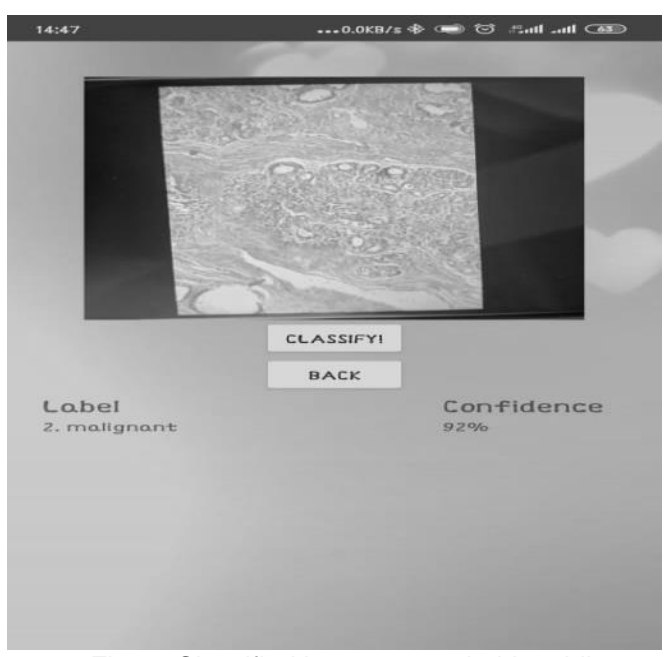

Fig. 5: Classified image on android mobile

\section{Conclusion}

In the context of classification, deep convolutional neural networks (CNNs) have been widely proven in the scientific and industrial community. In this work, we investigated the performance of a deep neural network model on a classification task related to breast cancer detection. The modification applied to the DenseNet model proves that deep learning model used in natural images processing can achieves high performance in medical images processing. In our case we achieve about $96 \%$ accuracy on test dataset and a $98 \%$ accuracy on training dataset in the breast cancer classification task.

\section{REFERENCES}

Ahmad, H. M., Ghuffar, S., \& Khurshid, K. (2018). "Classification of Breast Cancer Histology Images Using Transfer Learning", Department of Space Science Institute of Space Technology, Islamabad, Pakistan, 3(9), 119-128.

Bayramoglu, N. (2016). “Deep Learning for Magnification Independent Breast Cancer Histopathology Image Classification", Center for Machine Vision and Signal Analysis, University of Oulu, 6(2), 2-7

Chiao, J., Chen, K., Liao, K. Y., Hsieh, P., Zhang, G., \& Huang, T. (2019). "Detection and classi fication the breast tumors using mask R-CNN on sonograms". Medicine, Vol. 98, no. 19, pp. 1-5.

Cireşan, D. C., Giusti, A., Gambardella, L. M., \& Schmidhuber, J. (2013). "Mitosis detection in breast cancer histology images with deep neural networks". Lecture Notes in Computer Science, National University of Sciences and Technology Islamabad, Pakistan, pp. 411-418

Han, Z., Wei, B., Zheng, Y., Yin, Y., Li, K., \& Li, S. (2017). "Breast Cancer Multi-classification from Histopathological Images with Structured Deep Learning Model", International Journal of Advanced Computer Science and Applications 7(5), 16-22.

Irshad, H., Member, S., Veillard, A., Roux, L., \& Racoceanu, D (2014). "Methods for Nuclei Detection, Segmentation, and Classification in Digital Histopathology", International Journal of Computer Science \& Information Technology (IJCSIT), 10(2).
Kumar, S. S., Prateek, A., \& Vishu, M. (2016). "Breast Cancer Diagnosis using Digital Image Segmentation Techniques", International Journal of Advanced Computer Science and Applications, 6(3), 1-5.

LeCun, Y. (2001) "Comparison of learning algorithms for handwritten digit recognition", International Journal of Advanced Computer Science and Applications 1(2), 116-122

Lévy, D., \& Jain, A. (2016). Breast Mass Classification from Mammograms using Deep Convolutional Neural Networks, Stanford University, California 3(4), 295-302

Matthew, T.F, (2018) "Evaluation of computer-aided detection and diagnosis systems", Lombardi Comprehensive Cancer Center, Georgetown University, 3900 Reservoir Road, Northwest, Washington, DC, 23(4), 155-162.

Nawaz, M., Sewissy, A. A., \& Soliman, T. H. A. (2018). "Multi-Class Breast Cancer Classification using Deep Learning Convolutional Neural Network", International Journal of Advanced Computer Science and Applications 8(6), 316-322.

Ogundipe. S (2017). WHO urges early detection of cancer, as 80,000 die yearly in Nigeria Retrieved from https://www.vanguardngr.com/2017/02/urges-early-detectioncancer-80000-die-yearly-nigeria/

Olanfenwa, J (2019). Deploying Keras models to Android using Tensorflow. Retrieved from https://heartbeat.fritz.ai/deploying-pytorch-and-keras-modelsto-android-with-tensorflow-mobile-a16a1fb83f2

Platania, R., Lee, K., \& Park, S. (2018). “Automated Breast Cancer Diagnosis Using Deep Learning and Region of Interest Detection", International Journal of Advanced Computer Science and Applications Vol. 8, no. 5, pp. 218-225

Spanhol, F. A., Oliveira, L. S., Petitjean, C., \& Heutte, L. (2016). “A Dataset for Breast Cancer Histopathological Image Classification", Federal University of Parana Department of Informatics (DInf) Curitiba, PR - Brazil. Retrieved from https://web.inf.ufpr.br/vri/databases/_ breast-cancerhistopathological-database-breakhis

Spanhol, F. A., Oliveira, L. S., Petitjean, C., \& Heutte, L. (2017). Breast Cancer Histopathological Image Classification using Convolutional Neural Networks, IEEE Transactions on Biomedical Engineering, Vol. 63, no. 7, pp. 1455-1462.

Tao. Y, S. Lo, M.T. Freedman \& J. Xuan, (2007) A preliminary study of content-based mammographic masses retrieval. In Medical Imaging, 65141Z. International Society for Optics and Photonics. 